\title{
Spectropolarimetry in a sunspot penumbra
}

\section{Spatial dependence of Stokes asymmetries in Fe I $1564.8 \mathrm{~nm}$}

\author{
R. Schlichenmaier ${ }^{1}$ and M. Collados ${ }^{2}$ \\ 1 Kiepenheuer-Institut für Sonnenphysik, Schöneckstr. 6, 79104 Freiburg, Germany \\ 2 Instituto de Astrofísica de Canarias, 38200, La Laguna, Tenerife, Spain \\ e-mail: mcv@ll.iac.es
}

Received 17 September 2001 / Accepted 18 October 2001

\begin{abstract}
Stokes profiles of sunspot penumbrae show distinct asymmetries, which point to gradients in the velocity field and in the magnetic field. We present spectropolarimetric measurements of the Stokes vector in the neutral iron triplet at $1564.8 \mathrm{~nm}$ taken with the Tenerife Infrared Polarimeter (TIP) at the German Vacuum Tower Telescope (VTT) in Tenerife. We report on the peculiarities of the profiles of circularly and linearly polarized light for spots at different heliocentric angles. We elaborate on the spatial dependence of Stokes asymmetries within the penumbra and find for profiles of circularly polarized light: (1) In the center-side penumbra the amplitude difference of Stokes- $V$ exhibits a sign reversal on a radial cut, i.e., in the inner (outer) penumbra the red (blue) lobe is broader and shows a smaller amplitude than the blue (red) lobe. (2) In the outer limb-side penumbra (beyond the magnetic neutral line) the red lobe is broader and of less amplitude than the blue lobe. (3) Along the magnetic neutral line we find abnormal Stokes- $V$ profiles, which consist of more than 2 lobes. This indicates the presence of two polarities. For small heliocentric angles abnormal profiles are also seen beyond the magnetic neutral line in the outer penumbra. (4) Maps of the net circular polarization have the tendency to be antisymmetric with respect to the axis that connects disk center with spot center. This finding is striking, because corresponding maps for Fe I 630.25 are symmetric. For linearly polarized profiles we extract the following features: (5) On the center-side penumbra at a heliocentric angle of $56^{\circ}$ a Doppler-shift as high as $5 \mathrm{~km} \mathrm{~s}^{-1}$ can be directly measured by the splitting of the $\pi$-component of the linearly polarized component. (6) In limbside penumbrae, the profiles of the $\pi$-component show the typical asymmetry properties of the Evershed flow as observed in Stokes- $I$ of magnetically insensitive lines. (7) In the outer center- and limb-side penumbrae the center of the $\pi$-component is blue-shifted relative to the zero-crossing of the $V$-profile. Motivated by the moving tube model of Schlichenmaier et al. (1998b), we construct simple model atmospheres featuring hot upflows and cool outflows and calculate corresponding synthetic $V$-profiles. These profiles are compared with our measured ones and with observed $V$-profiles in FeI630.25 from other authors. We find that the synthetic $V$-profiles can reproduce all essential characteristics of observed $V$-profiles for both lines.
\end{abstract}

Key words. sunspots - Sun: magnetic fields - Sun: infrared - Sun: photosphere - techniques: polarimetric techniques: spectroscopic

\section{Introduction}

The penumbra of a sunspot is the manifestation of oblique magnetic field in a convectively unstable stratification and shows a variety of phenomena on small scales. The most intriguing penumbral phenomenon, the photospheric Evershed effect, is a line shift in Stokes- $I$ (Evershed 1909), which is proportional to wavelength (St. John 1913). These measurements indicated that the line shift is due to a Doppler-shift that corresponds to a horizontal outflow within the penumbra. In the 1960's it was established

Send offprint requests to: R. Schlichenmaier, e-mail: schliche@kis.uni-freiburg.de that the line shift is accompanied by a line asymmetry, such that the line wings are stronger shifted than the line core. The effect is often also referred to as line flags, line kinks, or line satellites (Bumba 1963; Servajean 1961; Holmes 1963; Maltby 1964; Schröter 1965). The line shift decreases, whereas the line asymmetry increases with formation height (Stellmacher \& Wiehr 1980; Ichimoto 1987, 1988; Degenhardt \& Wiehr 1994; Wiehr 1995; Balthasar et al. 1997). This observational finding can readily be explained with thin flow channels that lie in the deep photosphere (Maltby 1964; Rimmele 1995; Schlichenmaier et al. 1998a,b): lines that form deeply are shifted as a whole leading to a large line shift, but for lines that form in 
higher layers, only the line wing gets shifted leading to a small line core shift and a large asymmetry. This interpretation implies flow channels that correspond to magnetic flux tubes which are roughly horizontal and which are embedded in a steeper, non-moving background magnetic field. We note that discontinuities, or strong gradients, in the velocity and magnetic field arise when the line-of-sight (LOS) intersects the flux tube, i.e., the flow channel.

Not only the penumbral $I$-profiles, but also the profiles of $V, Q$, and $U$ exhibit asymmetries and Doppler shifts (e.g., Kjeldseth-Moe 1967; Beckers \& Schröter 1969; Grigorjev \& Katz 1972; Makita 1979). These Stokes profile asymmetries manifest the fine structure of a sunspot penumbra and carry information about gradients along the LOS in the velocity, the magnetic field strength and the inclination of the magnetic field vector (see, e.g., Sanchez Almeida \& Lites 1992, hereafter: SL92). SL92 classified the observed asymmetries of Stokes profiles by analyzing their spatial distribution within the sunspot, the profile shapes, and the amount of asymmetry. Their work was based on spectropolarimetric measurements of the magnetic line Fe I 630.25. This line is a Zeeman triplet with a Landé factor of 2.5 and its line core forms in the mid photosphere $(\log \tau \approx-2)$.

Since we expect the flow channels to reside in the deep photosphere, observations of an absorption line that forms as deeply as possible will provide us more constraints for theoretical models. In the present work we therefore use the infrared iron triplet at $1564.8 \mathrm{~nm}$ with a Landé factor of 3 . Its line core forms in the deep photosphere $(\log \tau \approx-1)$. In Sect. 2 we give details about the observed data set. We describe the data reduction, and especially the correction we need to apply for the residual crosstalk in Sect. 3. Section 4 is devoted to the systematic investigation of linearly and circularly polarized profiles. In particular we discuss the dependence of the shape of Stokes profiles on the location within the penumbra and on the heliocentric angle of the corresponding penumbra. In Sect. 5 we present simplified calculations of synthetic line profiles on the basis of the moving tube model (Schlichenmaier et al. 1998b). We demonstrate that the moving tube model is capable to reproduce qualitatively the observed characteristics of Stokes asymmetries. The conclusion is presented in Sect. 6.

\section{Observations}

We have measured the Stokes parameters of the infrared iron line $(g=3$, triplet $)$ at $1564.8 \mathrm{~nm}$, using the Tenerife Infrared Polarimeter (TIP) (Collados 1999; Martínez Pillet et al. 1999) at the German Vacuum Tower Telescope (VTT) at the Observatorio del Teide (Tenerife). Using the VTT correlation tracker (Schmidt \& Kentischer 1995; Ballesteros et al. 1996), we obtained maps of all Stokes parameters of a sunspot by scanning the solar image with a step size of about $0.4^{\prime \prime}$ across the slit, and a pixel size of $0.4^{\prime \prime}$ along the slit. The length of the slit corresponds to $24 \mathrm{Mm}$ on the sun, and the slit width was
Table 1. Listing of data sets.

\begin{tabular}{lll}
\hline Date in 1999 & Spot \# & Heliocentric angles, $\theta\left[^{\circ}\right]$ \\
\hline 21 Sep.-28 Sep. & AR 8704 & $33,28,28,56,64,82$ \\
21 Sep.-30 Sep. & AR 8706 & $59,45,33,16,24,39,47,61$ \\
09 Nov.-11 Nov. & AR 8755 & $30,22,20$ \\
\hline
\end{tabular}

AR 8704: negative polarity, southern hemisphere AR 8706: positive polarity, northern hemisphere AR 8755: positive polarity, northern hemisphere

Definition: Positive polarity, if the blue $V$-lobe is positive.

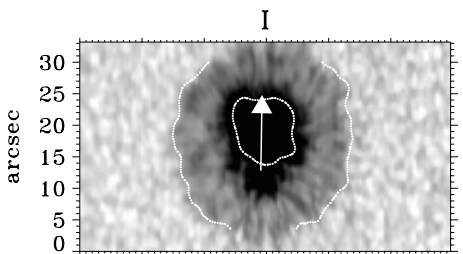

$|\mathrm{U}|$

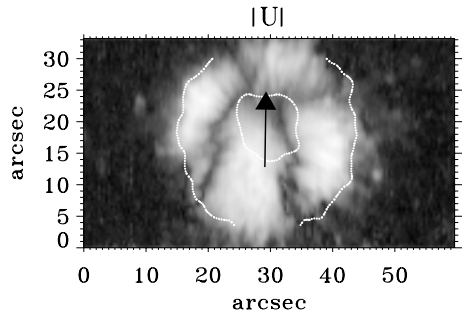

$|\mathrm{Q}|$

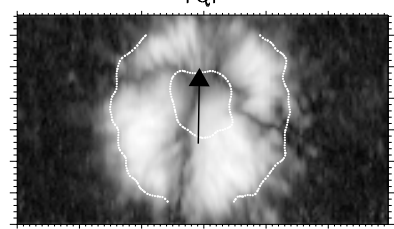

$|\mathrm{V}|$

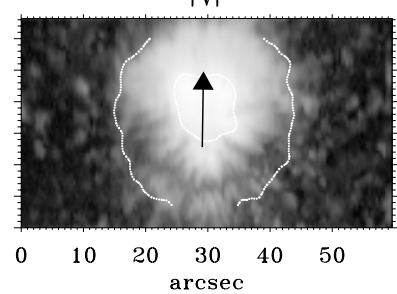

Fig. 1. Maps of the spectrally integrated Stokes profiles $\log (I)$, $\log |Q|, \log |U|$, and $\log |V|$ for the spot on Nov. 9 1999, at a heliocentric angle of $30^{\circ}$. The arrow points towards disk center.

$100 \mu \mathrm{m}$, corresponding to $0.5^{\prime \prime}$. In most of our maps the filamentary structure of the penumbra is visible and granules are resolved. From that we estimate a spatial resolution of about $1^{\prime \prime}$ for most of our maps. We achieve a polarimetric accuracy of some $2 \times 10^{-3}$ and a spectral resolution of $6 \mathrm{pm}$. We have observed 3 different sunspots at various heliocentric positions on the disk (see Table 1).

The chosen line forms in the deeper part of the photosphere. The line depression contribution function of the line core peaks around $\log \tau=-1$ for a quiet sun atmosphere, and originates significantly deeper than, e.g., the iron line at $630.25 \mathrm{~nm}$ for which the line core forms above $\log \tau=-2$ (for a comparison see, e.g., Fig. 2c of Rüedi et al. 1998). Therefore the IR line is better suited for a comparison of observations with predictions of the moving tube model.

For the spot on Nov. 9 1999, we display in Fig. 1 the intensity map $\left(\int I(\lambda) \mathrm{d} \lambda\right)$, and the spectrally integrated maps of absolute polarization, e.g., $\int|V(\lambda)| \mathrm{d} \lambda$. We mention typical features of these maps: (1) The maps of $|Q|$ and $|U|$ show four quadrants being rotated against each other by 45 degrees. This is the consequence of a radial component of the magnetic field which revolves around the center of the spot. (2) The $|V|$-map is to a large degree symmetric with respect to the line that connects disk center and spot center (axis-of-symmetry). (3) On the limbside penumbra of the $|V|$-map, a curved line of minimal 
$|V|$-signal is apparent, which is called the magnetic neutral line. We come back to the significance of the axis-ofsymmetry and the magnetic neutral line in Sect. 4.

\section{Calibration and cross-talk correction}

As described by Collados (1999) and Martínez Pillet et al. (1999), TIP uses a polarization modulator formed by a pair of ferro-electric liquid crystals (FLCs) followed by a calcite beam-splitter. The FLCs behave as retarders with fixed retardance, whose axes can be switched between two positions, depending on an externally supplied voltage. In this case, the two FLCs have retardances near 180 and 90 degrees and the switching angle is about $50^{\circ}$. This value is temperature dependent (with a variation of $\sim 0.3^{\circ} /{ }^{\circ} \mathrm{C}$ ) and necessitates a temperature control of the environment where the modulator package is located. We have checked that the temperature at the location of the FLCs is stable within $0.5{ }^{\circ} \mathrm{C}$ and that there is no consequence on the stability of the recovered Stokes parameters.

The frame rate is $8 \mathrm{~Hz}$. One FLC is modulated at $4 \mathrm{~Hz}$ and the other at $2 \mathrm{~Hz}$. As a consequence, every $0.5 \mathrm{~s}$, four different linear combinations of the input Stokes parameters are measured and the full Stokes vector at all wavelengths and at all points along the slit is obtained. As explained by Collados (1999), the two beams produced by the calcite are combined to reduce the seeing-induced crosstalk (i.e., the false polarization signals due to the temporal variation of the observed Stokes parameters at temporal scales below $0.5 \mathrm{~s}$ ). The cycle is repeated and images are accumulated until the desired $\mathrm{S} / \mathrm{N}$ ratio is achieved. Typically, values of $2 \times 10^{-3}$ (one standard deviation in the continuum of $Q, U$, and $V$ in units if the continuum intensity) are achieved with a total integration time of $5 \mathrm{~s}$. In the following, the most important corrections applied to the data are described.

\subsection{Flat fielding}

As usual, the detector pixel-to-pixel differential response has to be removed by a flat fielding process. To that aim, images are taken in quiet regions while moving the telescope. With a modulation frequency of $2 \mathrm{~Hz}$ (as described above), 500 images are averaged at every modulation step. This procedure has turned out to be very efficient to "defocus" the spatial structures on the solar surface. The average spectral profile is used to remove the spectral line at every row of the average image.

The flat fielding process has two further motivations. On the one hand, the response of the detector is characterized by the presence of a large amplitude interference fringe pattern. Fortunately, this pattern (although dependent on wavelength) is very stable in time and does not leave any signature on the polarization spectra. It is easily removed from the intensity images by the flat field procedure. On the other hand, there is a second set of interference fringes generated by the FLCs, which are not stable in time and move along the detector. These fringes are polarized and show up in the polarization images with amplitudes of the order of one percent of the intensity image. A linear interpolation between two flat field images, separated in time by not more than two hours, is enough to remove these fringes below the noise.

\subsection{Calibration of the polarization modulator}

The particular behavior of the polarimeter at the observed wavelength is calculated after generating light beams of known polarization states. Herefore, calibration optics are inserted at an appropriate position in the beam. The calibration optics consists of a linear polarizer (which can be oriented at any angle but is kept fixed during the acquisition of calibration data) and of a retarder (very similar to a quarter-wave plate at the wavelength considered in this paper). This latter element is rotated a full turn, while data is taken (in the same way as with normal data) at steps of five degrees. The matrix relating the input Stokes parameters and the observed intensities, the modulation matrix, is derived from a least-squares fit. Due to the special location of the calibration optics, the modulation matrix not only takes into account the behavior of the modulation package, but also all the optical elements between the polarimeter and the calibration optics. In particular, it includes several mirrors which form the correlation tracker unit. The inverse of that matrix, the demodulation matrix, is used to calculate the unknown input Stokes parameters from the measured intensities at the detector.

\subsection{Müller matrix of the telescope}

The modulation matrix does not correct for the cross-talk introduced by the telescope itself and the coelostat which folds the light into it. At the time of the observations we did not have at our disposal any large infrared polarizing sheet which could be used to derive the behavior of these big optical elements (with diameters of $70 \mathrm{~cm}$ ). A geometrical model of the VTT has been constructed following Capitani et al. (1989), and refractive indices for aluminum are taken from the standard table in Lide (1993). An example of a daily variation of the Müller matrix of the telescope is shown in Collados (1999).

\subsection{Correction of residual cross-talk}

Our model of the coelostat and the telescope has a restricted validity, because the refractive indices of the reflecting layers are standard values taken from the literature, and may well differ from the actual values, especially for the coelostat mirrors which are in open air. Therefore, a certain amount of residual cross-talk is typically present in our calibrated Stokes profiles. We correct for it using a statistical method which takes advantage of the geometrical properties of the sunspot magnetic field. Our method is similar to the one used by SL92 in their Sect. 2 . 
The residual cross-talk from $I$ to $Q, U$, and $V$ is removed requiring that no continuum polarization is present in the polarized profiles. Averaging the map of a sunspot we determine the mean cross-talk terms and subtract the corresponding fractions of $I(\lambda)$ from $Q(\lambda), U(\lambda)$, and $V(\lambda)$. To disentangle the mixing between the linear and circular polarization, we take into account that $Q$ and $U$ change sign four times along an azimuthal circle within the penumbra, while the sign of $V$ only changes across the magnetic neutral line. Thus, except for spots very near the limb, we expect a negligible correlation between linear and circular polarization. According to this reasoning, we have applied the following two steps to every sunspot observation:

1. We have selected those spectral profiles where the maximum absolute value of $V(\lambda)$ is at least 5 times larger than the maximum absolute values of $Q(\lambda)$ and $U(\lambda)$. For spots with a moderate heliocentric angle, these profiles are mainly located in the umbra, where the magnetic field is preferentially longitudinal. Two linear fits $Q(\lambda)=C_{V Q} V(\lambda)$, and $U(\lambda)=C_{V U} V(\lambda)$ are performed. The resulting coefficients $C_{V Q}$ and $C_{V U}$ are interpreted as the residual cross-talk from $V$ to $Q$ and $U$, respectively. The "true" linear polarization profiles are then evaluated, at every observed point, as

$Q_{\text {true }}(\lambda)=Q(\lambda)-C_{V Q} V(\lambda)$.

An equivalent equation is applied on $U$. It is worthwhile noting that the least-squares fit equations give

$C_{Q U}=\sum_{i, j} Q\left(\lambda_{j}\right) V\left(\lambda_{j}\right) / \sum_{i, j} V^{2}\left(\lambda_{j}\right)$,

(and an equivalent one for $C_{V U}$ ). The index $i$ runs over all the selected points, and index $j$ represents the discrete set of wavelengths of the observed spectra. Equation (2) is the same as that used by SL92 to evaluate the cross-talk coefficients from their sunspot observations, although it is not clear to us whether they performed the correction as expressed in Eq. (1).

2. A similar procedure is applied to evaluate the crosstalk from linear to circular polarization. We have selected those profiles where the maximum absolute values of $Q$ or $U$ are at least 5 times larger than the maximum absolute value of $V$. Predominantly, these profiles are located near the magnetic neutral line. For the deep forming FeI1564.8, the magnetic neutral line is well within the penumbra for heliocentric angles larger than some $15^{\circ}$. Therefore, for the heliocentric angles that we consider a significant number of profiles are selected (this was not the case for SL92). For the selected profiles we perform a linear fit, $V(\lambda)=C_{Q V} Q(\lambda)+C_{U V} U(\lambda)$. The parameters $C_{Q V}$ and $C_{U V}$ represent the residual crosstalk from $Q$ and $U$ to $V$, respectively. $V$ is corrected at every observed point according to the corresponding version of Eq. (1). We have verified that the same results are obtained if we make the calculations separately for $Q$ and $U$.
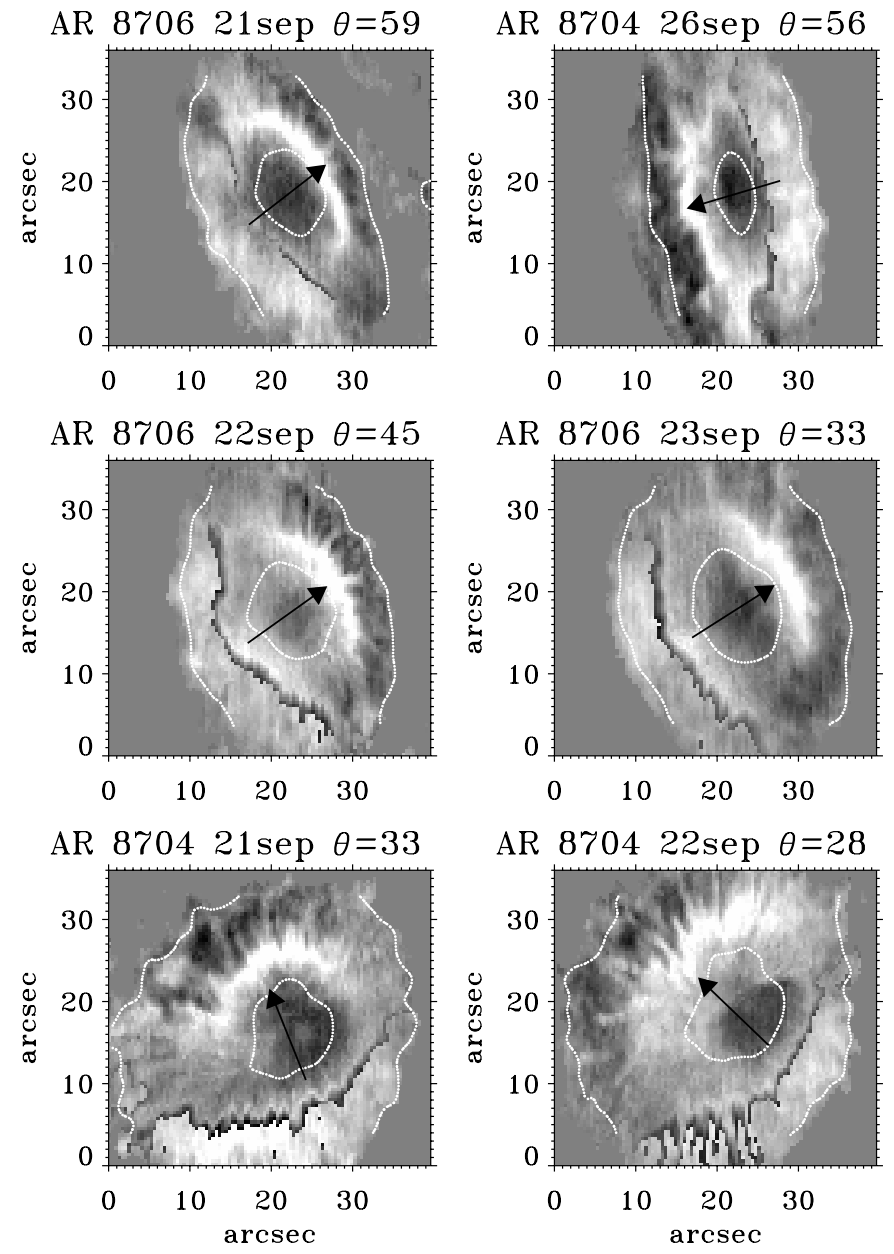

AR 8704 22sep $\theta=28$
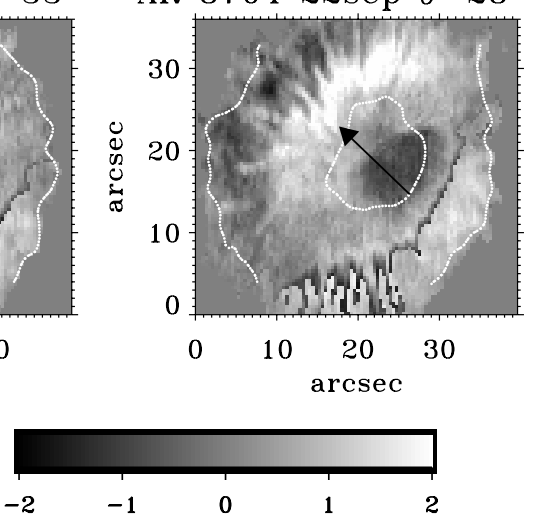

Fig. 2. Amplitude difference of the $V$-profile, $\mathrm{d} a=a_{\mathrm{b}}-a_{\mathrm{r}}$. The spot number, the date, and the heliocentric angle, $\theta$, are given above each map. The arrow points towards disk center and corresponds to the axis-of-symmetry. The contours mark the inner and outer boundaries of the white-light penumbra. The gray-scale coding is in units of $V$ as given in Eq. (4).

After these corrections have been applied, we feel confident that any residual crosstalk is below our noise level, and thus, does not contaminate the results presented in the next sections.

\section{Profile asymmetries within the penumbra}

The penumbral fine structure consists of small-scale variations of the flow field and of the magnetic field. Measurements of Stokes- $I$ asymmetries are well suited to diagnose lateral and depth variations of the flow field. In order to diagnose variations of the flow field and the magnetic field, it is more appropriate to measure the full Stokes vector. To describe the degree of asymmetry of a certain $V$-profile, we introduce the amplitude difference, $\mathrm{d} a$, and the net circular polarization, NCP. 
Definitions: The amplitude differences, $\mathrm{d} a$, of a $V$-profile is defined as

$\mathrm{d} a \equiv\left|a_{\mathrm{b}}\right|-\left|a_{\mathrm{r}}\right|$,

where $a_{\mathrm{b}}$ is the amplitude of the blue lobe of $V(\lambda)$ and $a_{\mathrm{r}}$ correspondingly for the red lobe. The sign of $\mathrm{d} a$ does not depend on the polarity of $V(\lambda)$, since the amplitudes enter with absolute values. Note that we normalize $V(\lambda)$ relative to the corresponding continuum, and give its value in units of percent, i.e.,

$V(\lambda) \equiv 100 \cdot \frac{V_{\text {measured }}(\lambda)}{I_{\mathrm{c}}}$.

Since abnormal $V$-profiles with more than two lobes are also present in the penumbra, the definitions of $a_{\mathrm{b}}$ and $a_{\mathrm{r}}$ are generalized as follows. The wavelength positions of the maximum and the minimum of the profile, $\lambda_{\max }$ and $\lambda_{\min }$, are used to define the amplitudes:

$a_{\mathrm{b}} \equiv\left\{\begin{array}{ccc}\max (V(\lambda)) & \text { for } \lambda_{\max }<\lambda_{\min } \\ |\min (V(\lambda))| & \text { for } \lambda_{\min }<\lambda_{\max }\end{array}\right.$

$a_{\mathrm{r}} \equiv\left\{\begin{array}{c}|\min (V(\lambda))| \text { for } \lambda_{\max }<\lambda_{\min } \\ \max (V(\lambda)) \text { for } \lambda_{\min }<\lambda_{\max }\end{array}\right.$.

The net circular polarization, NCP, is defined through the spectral integration over FeI $1564.8 \mathrm{~nm}$,

$\mathrm{NCP} \equiv \int V(\lambda) \mathrm{d} \lambda$.

Axis-of-symmetry: If one assumes that a sunspot is axially symmetric and that it has no azimuthal component, the line that connects disk-center and spot-center forms an axis-of-symmetry, i.e., a map of any physical quantity, like, e.g., the magnetic field strength, is symmetric with respect to this axis. Indeed, at photospheric level observed circular sunspots are roughly axially symmetric at least for their global appearance, and azimuthal components of the flow and magnetic field are negligibly small. On small scales the penumbral fine structure introduces an azimuthal dependence, but not an azimuthal component. Since the penumbral flow field is predominantly radial, the LOS component of the flow field is expected to be largest along the axis-of-symmetry, i.e., the observed asymmetries are expected to be largest along the axis-of-symmetry.

Magnetic neutral line (MNL): For an axially symmetric sunspot, one observes a MNL which is symmetric with respect to the axis-of-symmetry. The MNL stems from the fact that the mean magnetic field along this line is perpendicular to the LOS, such that its Stokes- $V$-signal vanishes. But any fine structure magnetic field that has an inclination different from the mean magnetic field should produce a signal, and indeed it is found that the Stokes$V$-profiles along the neutral line do not completely vanish and that the residual profiles are to a high degree abnormal. That means that $V$-profiles along the MNL are ideally suited to diagnose the imprints of the penumbral fine structure. However, the $V$-profiles along the MNL can be spoiled by two instrumental effects: (1) Along the MNL the $Q$ - and $U$-profiles have much larger amplitudes than the $V$-profiles and hence the $V$-profiles are susceptible to cross-talk from $Q$ and $U$. (2) Stray-light from the vicinity of the MNL, where the $V$-amplitude is larger may also effect the $V$-profiles along the MNL.

Maps of amplitude difference: Figure 2 contains gray-coded maps of the amplitude difference for a number of observed spots at different heliocentric positions. Details of the amplitude differences are discussed in the next subsections. At this point we like to give an overview of a few general features of these maps: (1) The axes-of-symmetry (extensions of the arrows in the figure) represent axes with a high degree of symmetry, meaning that the azimuthal variation of $\mathrm{d} a$ within the spot is rather small. (2) Along a radial cut on the center-side penumbra the amplitude difference exhibits a sign reversal. (3) On the outer limb side, beyond the MNL, the amplitude difference has the opposite sign as in the outer disk-center-side penumbra. (4) The MNL is apparent as a sharp line of negative amplitude difference.

In the following subsections we classify the observed asymmetries of Stokes profiles along the axis-of-symmetry and along the MNL. We distinguish between the centerside (Sect. 4.1) and the limb-side penumbra (Sect. 4.2). The net circular polarization is discussed in Sect. 4.3.

\subsection{Profiles on the disk-center-side}

It is apparent in Fig. 2 that the amplitude difference shows a sign reversal on a radial cut of the center-side penumbra. For a closer inspection of the Stokes profiles, Fig. 3 displays the profile shapes along a radial cut from the inner (bottom) to the outer (top) center-side penumbra (AR 8704 at $\theta=56^{\circ}$, upper right panel in Fig. 2). The left column shows $V(\lambda)$ (thick line) and the total linear polarization,

$L(\lambda) \equiv \sqrt{Q(\lambda)^{2}+U(\lambda)^{2}}$

(thin line). Since $L$ is much smaller than $V$ on the centerside penumbra, $L$ is multiplied by a factor of 4 for better visibility. The right column displays the total polarization,

$P(\lambda) \equiv \sqrt{Q(\lambda)^{2}+U(\lambda)^{2}+V(\lambda)^{2}}$.

(thick line) and again $L(\lambda)$. All quantities are given as percentage of the corresponding continuum intensity (cf. Eq. (4)). The numbers next to the $V$-profiles give the amplitude difference of $V(\lambda)$, and the arrows illustrate the corresponding signs and magnitudes. The length of the arrows is a factor of 5 larger than the scale on the $y$-axis. For this scan we point out a number of peculiarities:

1. As illustrated by the arrows and the numbers, the amplitude difference changes sign along the radial cut. In the inner penumbra $\mathrm{d} a$ is positive with a maximum 

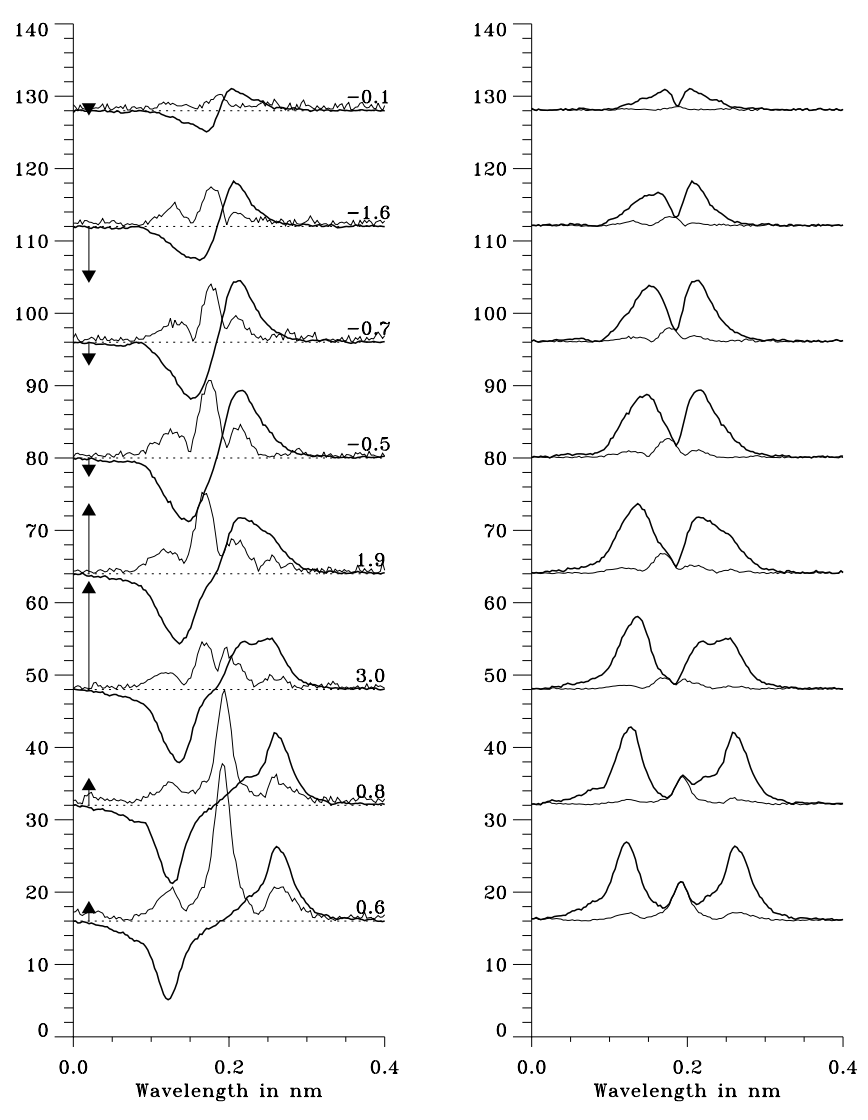

$\longmapsto 10 \mathrm{~km} / \mathrm{s}$

$\longmapsto$ line splitting of 1000 Gauss

Fig. 3. Profiles along a radial cut from the inner (bottom) to the outer (top) center-side penumbra for a AR 8704 at $\theta=56^{\circ}$. The left column shows $V(\lambda)$ (thick line), and $L(\lambda)$ (thin line, cf. Eq. (8)). Since the mean field is predominantly along the LOS, $L$ is much smaller than $V$, and is multiplied by a factor of 4 for better visibility. The right column shows $P(\lambda)$ (thick line) and $L(\lambda)$ (thin line). All quantities are given in percent of the corresponding continuum intensity. The numbers next to the profiles in the left column denote the amplitude difference, the arrows illustrate the sign and magnitude (multiplied by a factor of 5) of the amplitude differences. The two bars at the bottom indicate the wavelength scales of a Doppler shift of $10 \mathrm{~km} \mathrm{~s}^{-1}$ and a splitting of the $\sigma$ components for 1000 Gauss, respectively.

of 3.0. The corresponding $V$-profile shows that the red lobe is broader and of smaller amplitude than the blue lobe. In the outer penumbra this behavior reverses. There, the blue lobe is broader and of smaller amplitude than the red lobe, and $\mathrm{d} a$ is negative with an extremum at -1.6 .

2. The lobes of $L(\lambda)$ show a fascinating behavior along the scan: The $L$-profiles near the bottom and near the top of Fig. 3 have a normal shape, i.e., they have a central $\pi$-component and two Zeeman-shifted $\sigma$ components. Following the $\pi$-component along the scan from bottom (inner penumbra) to top (outer penumbra), one notices that the $\pi$-component from the bottom does not lead into the $\pi$-component at the top. Instead, the $\pi$-component decreases gradually in
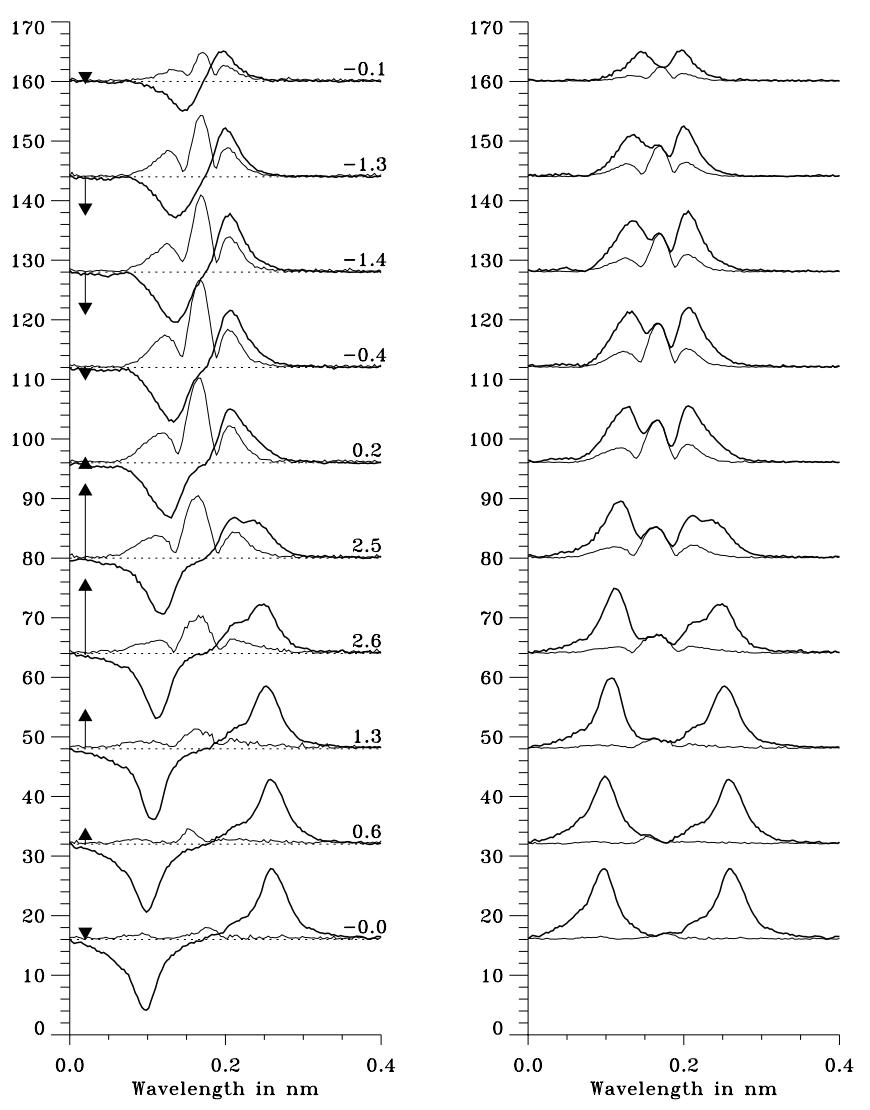

$\longmapsto 10 \mathrm{~km} / \mathrm{s}$

$\longmapsto$ line splitting of 1000 Gauss

Fig. 4. Same as Fig. 3. For the same spot (AR 8704), but at a smaller heliocentric angle, $\theta=33^{\circ} . L(\lambda)$ is multiplied by a factor of 2 (instead of 4 ) in the left column.

amplitude as one goes upwards, and a second blueshifted $\pi$-component develops, which gradually increases in amplitude and which merges in the $\pi$ component at the top. At the point of largest positive $V$-amplitude difference (where the zero level of the profiles is at 48 on the $y$-axis), the two $\pi$-components have similar amplitudes. The Doppler-shift of the two $\pi$-components for that profile corresponds to a velocity of approximately $5 \mathrm{~km} \mathrm{~s}^{-1}$.

3. In the outer penumbra, it is seen that the peak of the $\pi$-component in $L$ is blue-shifted relative to the zerocrossing of the $V$-profile. This shift corresponds to a Doppler-velocity of approximately $1.5 \mathrm{~km} \mathrm{~s}^{-1}$.

In order to work out the dependency of these peculiarities on $\theta$, we show in Fig. 4 the corresponding profiles of the same spot as in Fig. 3 at a smaller heliocentric angle of $\theta=33^{\circ}$. From this and our other data sets (cf. Table 1), we find that the sign reversal of the amplitude difference (point 1 of the upper list) is present at all heliocentric angles, even though the amplitude difference in the outer penumbra slightly decreases with smaller heliocentric angles. Points 2 and 3 of the upper list are dependent on the heliocentric angle. We see this behavior for spots with a heliocentric angle that exceeds $\theta \approx 50^{\circ}$. For spots closer to disk center, the $\pi$-component of the inner 
penumbra gradually merges with the $\pi$-component of the outer penumbra, as it can be seen in Fig. 4. Also, in the outer penumbra of spots with small $\theta$ the wavelength-shift between the peak of the $\pi$-component in $L$ and the zerocrossing of $V$ diminishes. It seems obvious that a horizontal flow component is responsible for the peculiarities in points 2 and 3 , since the projection of the LOS component of a horizontal flow decreases as a function of $\sin \theta$, such that the blue-shifted $\pi$-component in the outer penumbra would disappear for a small $\theta$. We discuss the observed profiles of linearly polarized light in Sect. 5.4.

Comparing our findings with previous work, we note that SL92 find negative Stokes- $V$ amplitude differences on the center-side penumbra, corresponding to our finding in the outer penumbra, but no sign reversal. They analyzed four spots at small heliocentric angles $(\cos \theta=0.97,0.96$, $0.90,0.80)$ and used the iron line at $630.25 \mathrm{~nm}$. A possible explanation for the sign reversal of the amplitude difference in FeI 1564.8 and the fact that it is not observed in Fe I630.2 is discussed in Sect. 5.1.3.

\subsection{Profiles on the limb-side}

In the limb-side penumbra the $V$ amplitudes are typically smaller than the $L$ amplitudes, since the mean magnetic field is predominantly perpendicular to the LOS. The magnetic neutral line (MNL, cf. Sect. 2) constitutes a special configuration: the mean magnetic field is to a large degree perpendicular to the LOS, but the fine structure, e.g., horizontal flow channels, have a large component along the LOS. It is therefore expected that $V$-profiles that stem from the MNL are dominated by imprints of the fine structure rather than by the background magnetic field. In this subsection we discuss profile shapes along a radial cut and along the MNL, and we distinguish between small and large heliocentric angles, $\theta . V$-profiles that exhibit more than two lobes are called abnormal.

\subsubsection{Profiles along a radial cut}

In Figs. 5 and 6 radial cuts on the limb-side penumbra of the spot AR 8706 are shown for $\theta=59^{\circ}$ and $\theta=$ $33^{\circ}$, respectively. For the large heliocentric angle, the MNL is located in the inner penumbra. The arrows aside each profile point in that direction. For $\theta=33^{\circ}$ the MNL lies in the middle penumbra. The $V$-profiles in the two figures show a complex behavior, even more complex than on the center-side penumbra:

1. The main and common feature in both figures is that the $V$-profile in the inner penumbra (bottom) has a positive polarity with a strong splitting and in the outer penumbra (top), the $V$-profiles exhibit the other polarity with a smaller splitting. The decrease of the splitting from the inner to the outer penumbra can readily be explained by a decrease of the magnetic field strength, while the polarity change of $V$ is due to an increase of the inclination of the mean magnetic field,
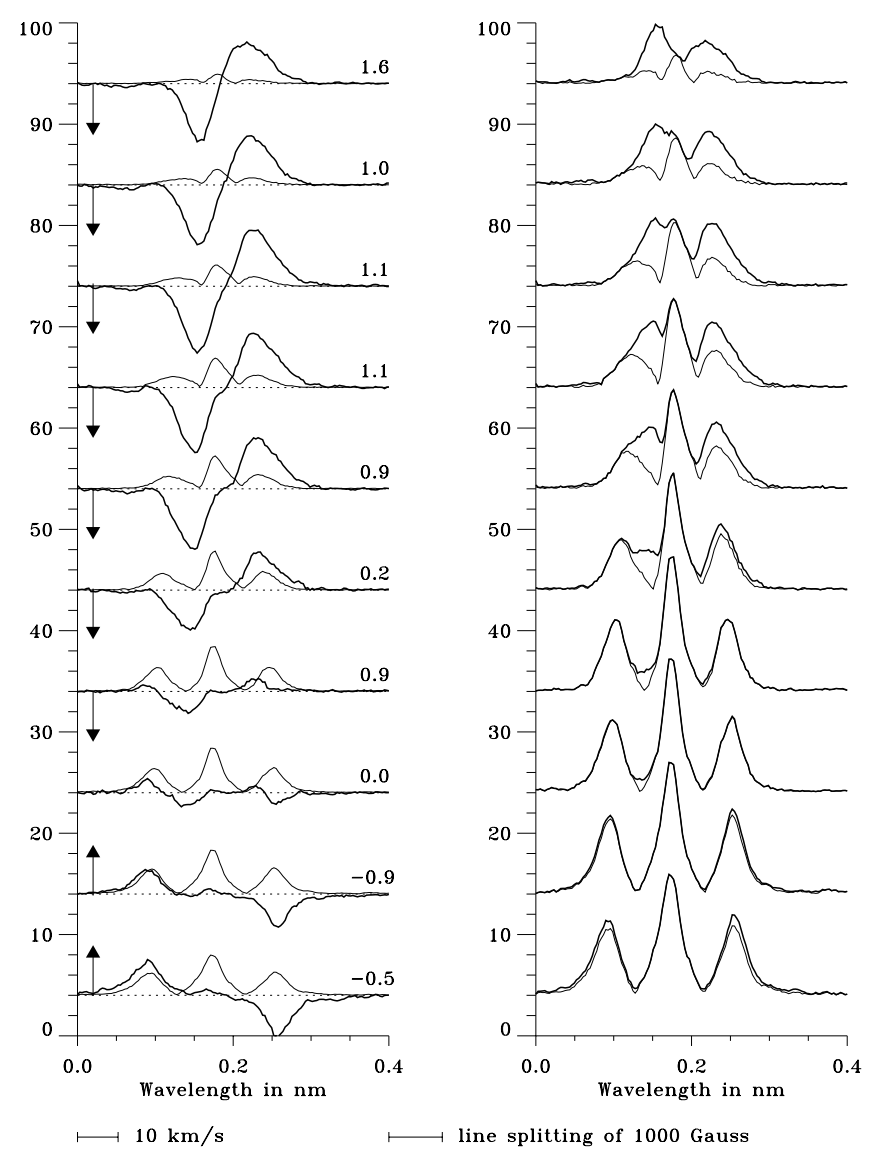

Fig. 5. Profiles along a radial cut from the inner (bottom) to the outer (top) limb-side penumbra for the spot AR 8706 at $\theta=59^{\circ}$. As in Fig. 3 the left column shows $V(\lambda)$ (thick line) and $L(\lambda)$ (thin line, divided by a factor of 4 ), and the right column shows $P(\lambda)$ and $L(\lambda)$. The arrows point toward the magnetic neutral line and the numbers next to each $V$-profile give the amplitude difference. Note that AR 8706 has the opposite polarity than AR 8704, which was used in Figs. 3 and 4 .

which changes polarity relative to the LOS. The transition of $V$-shapes from the inner to the outer penumbra is gradual and may be ascribed to a gradual decrease of one component, while the component of the other polarity gradually increases. At the MNL both components are of equal strength and, hence, the profile is due to two components of opposite polarity.

2. For $\theta=59^{\circ}$ (Fig. 5) the co-existence of the two components of opposite polarity, i.e., the presence of more than two lobes, is restricted to the location of the MNL. For the smaller heliocentric angle (Fig. 6) the blue lobe of a positive polarity component is seen almost all the way to the outer penumbra. We come back to this in Sect. 5.2.1.

3. For both heliocentric angles the amplitude difference in the outer limb-side penumbra has positive values, i.e., the red lobe is broader and of less amplitude than the blue lobe. For the outer center-side penumbra, $\mathrm{d} a$ is negative (cf. list item 1 of Sect. 4.1). In Sect. 5.2 we 


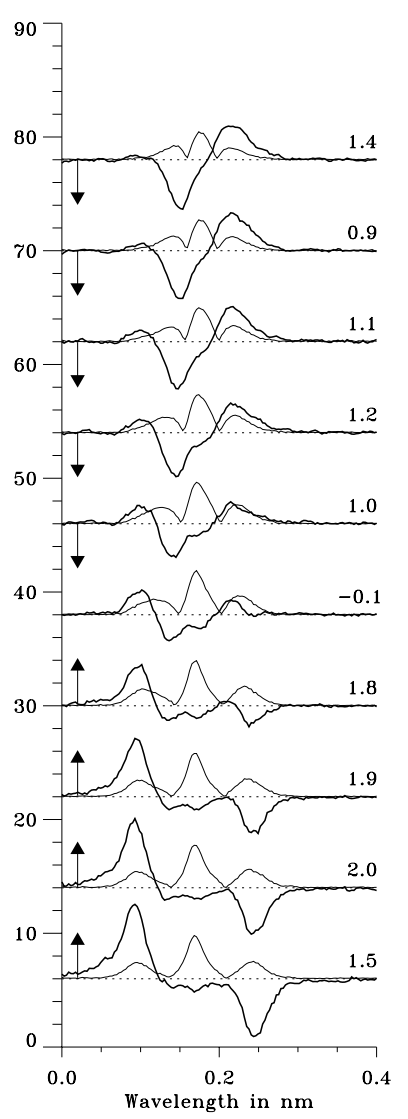

$\longmapsto 10 \mathrm{~km} / \mathrm{s}$

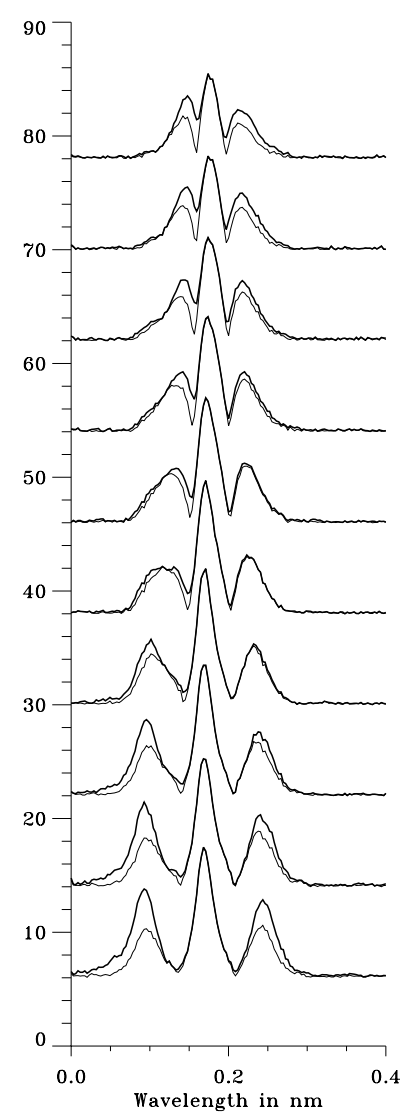

$\longmapsto$ line splitting of 1000 Gauss

Fig. 6. Same as Fig. 5, but for the same spot at a smaller heliocentric angle, $\theta=33^{\circ}$.

demonstrate that this behavior can be understood as a consequence of horizontal flow channels.

4. In the middle penumbra the $P$-profile exhibits a blue lobe that is strongly broadened relative to the red $P$-lobe (see profile at 44 on the $y$-axis in Fig. 5 and profile at 38 in Fig. 6 ). Tracing the $P$-profile from the middle to the outer penumbra one finds that the blue $P$-lobe is closer to the central lobe than the red $P$-lobe, i.e., the distance $\left|\sigma_{\mathrm{b}}-\pi\right|$ becomes smaller than $\left|\sigma_{\mathrm{r}}-\pi\right|$ as one approaches the outer penumbra. It seems that this behavior of $P$ can also be described by the finding, that the center of the $\pi$-component of $L$ is blue-shifted relative to the zero-crossing of the $V$-profile. This phenomenon is a general feature, largely independent of the heliocentric angle. It should also be noted that the $\pi$-component of $L$ displays the typical Evershed asymmetry (wings stronger shifted than the core) as observed in Stokes- $I$ of magnetically insensitive lines. A qualitative explanation of these peculiarities is given in Sect. 5.4.

\subsubsection{Profiles along the magnetic neutral line}

In this paragraph, we discuss $V$ - and $L$-profiles along the MNL of a spot at $\theta=33^{\circ}$ (Fig. 7). At this heliocentric angle, the MNL crosses the axis-of-symmetry in the middle of
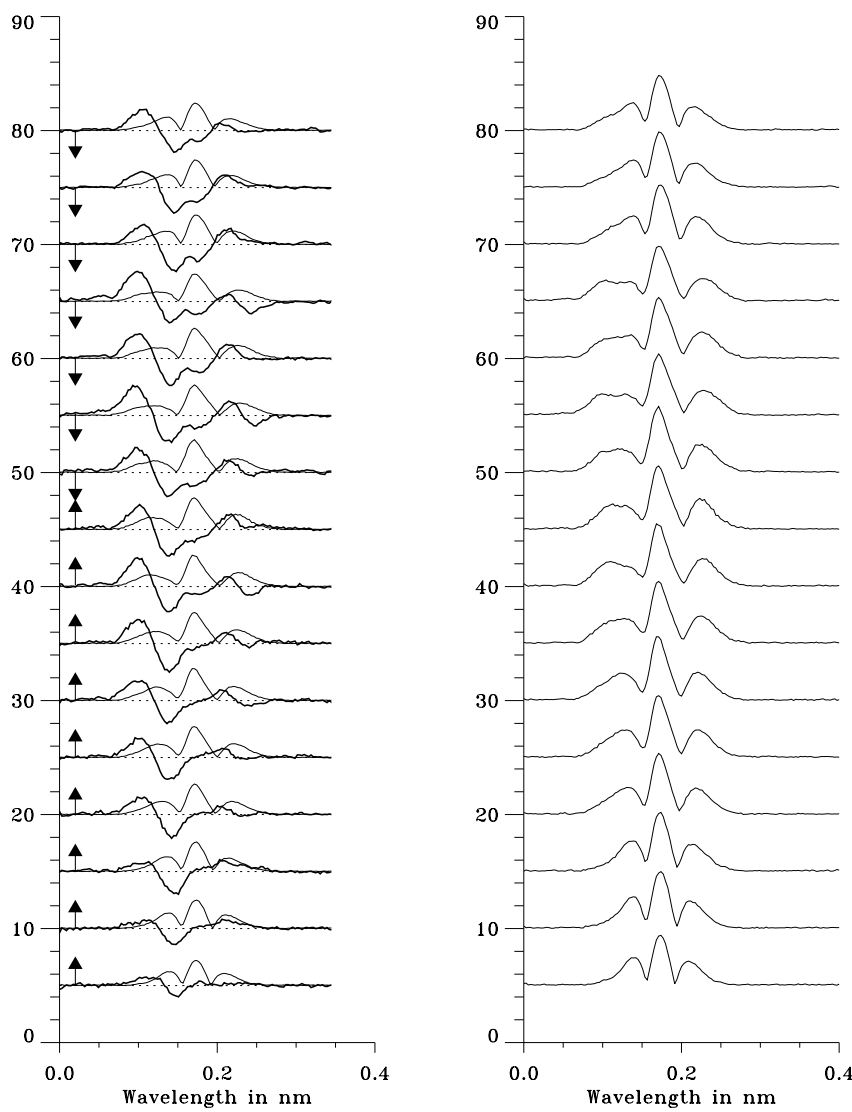

$\longmapsto 10 \mathrm{~km} / \mathrm{s} \quad \longmapsto$ line splitting of 1000 Gauss

Fig. 7. Profiles along the magnetic neutral line for the spot at $\theta=33^{\circ}$. The left column displays $V(\lambda)$ (thick line) and $L(\lambda)$ (thin line, divided by 2). Arrows point toward the position of the axis-of-symmetry. The right column shows $P(\lambda) / 2$.

the penumbra. In this figure the $V$-profiles typically have a negative amplitude difference, which reflects the sharp line of negative amplitude difference along the MNL in Fig. 2. Apart from that we find:

1. The $V$-profiles clearly consist of more than two lobes, hence are abnormal. This indicates that components of different polarities contribute to the line profile, and we discuss this in more detail in Sect. 5.3. Such abnormal $V$-profiles have been referred to as the cross-over effect (Grigorjev \& Katz 1972).

2 . The $\pi$-component of the linearly polarized light, $L(\lambda)$, is asymmetric near the axis-of-symmetry and becomes of more and more regular shape as the azimuthal distance increases. This is expected for a predominantly horizontal radial flow, since the LOS-component of the flow velocity is highest at the axis-of-symmetry.

\subsection{Net circular polarization (NCP)}

In our data set, the cross-talk that stems from the coelostat is corrected using a model of the telescope with standard refraction indices, instead of actually measuring these values. As a result, we deal with a small amount of residual cross-talk by correcting it with a statistical 


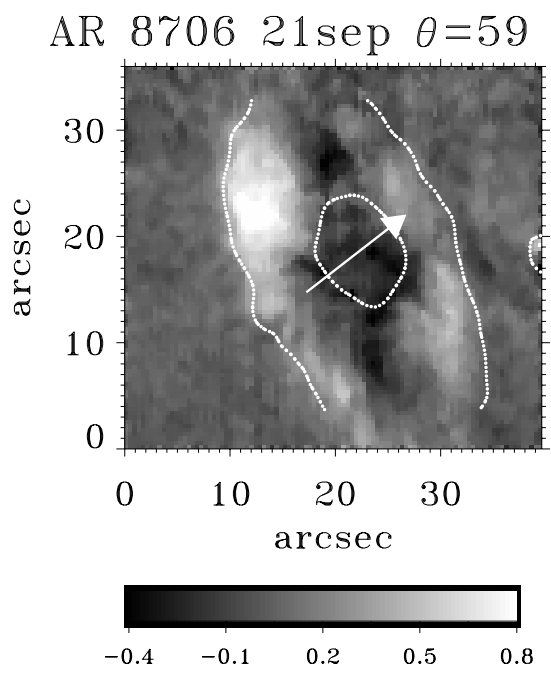

Fig. 8. Map of net circular polarization of a sunspot at $\theta=59^{\circ}$ for FeI 1564.8 (gray-scale coding is in units of pm). The data set corresponds to the upper left panel of Fig. 2.

method (see Sect. 3). Even though we are confident that our cross-talk correction is accurate enough so that the basic profile properties discussed in the preceeding sections are real, we are cautious about evaluating the NCP for our profiles. For the NCP already a small residual of cross-talk from $Q$ or $U$ to $V$ can lead to spurious results. Therefore, we limit our analysis to the spatial distribution of NCP, which we believe is free of spurious cross-talk effects.

From the iron line at $630.25 \mathrm{~nm}$ it is known that NCP maps of sunspots are roughly symmetrical relative to the axis-of-symmetry, which we defined in Sect. 4 (see, e.g., Westendorp Plaza et al. 2001). In contrast to this result, our NCP maps are to a large degree antisymmetric with respect to the axis-of-symmetry. As an example, Fig. 8 displays the NCP map for a spot at $\theta=59^{\circ}$. Since all our NCP maps of spots with an intermediate heliocentric angle show a similar behavior independent of the orientation of the axis-of-symmetry relative to the slit, we are confident that this is not an effect of cross talk.

Müller (2001) gives an explanation for this puzzling difference on the grounds of synthetic profiles that stem from flow channels which reside in the deep photosphere and are embedded in a background magnetic field. He investigates the variation of NCP depending on the azimuthal position within an axially symmetric model sunspot. He finds that if one switches off the magnetooptical effects, the NCP is strictly symmetric with respect to the axis-of-symmetry for any absorption line. However, including the magneto-optical effects introduces a strong antisymmetric contribution in the NCP map of the infrared triplet, while the NCP map of Fe I 630.25 does not change significantly. For an understanding of this behavior, one has to realize that the azimuthal angles with respect to the LOS of the flow channel, $\phi$, and the magnetic background field, $\phi^{\prime}$, change along an azimuthal circle within a sunspot. Discontinuities across flow channels that have a non-vanishing $\delta \phi=\phi-\phi^{\prime}$ produce NCP even if $|B|$ and $\gamma$ do not change along the LOS, but only if the magneto-optical effects are included in the calculation (see Landolfi \& Landi degl'Innocenti 1996). Hence, the difference between our NCP maps for FeI1564.8 and for Fe I 630.25 can be reconciled, if one assumes that outflow channels, which are more or less horizontal, are present in deep layers of the photosphere. The fact that the two NCP maps are so different is essentially due to the large wavelength difference between the two lines, because the Zeeman splitting which is proportional to $\lambda^{2}$ acts differently than the Doppler shift, which is proportional to $\lambda$.

\section{Synthetic spectral lines}

The large variety of systematic dependencies of Stokes profiles on position and wavelength pose severe constraints on models for the penumbra, which present models do hardly fulfill. Although inversion codes, like, e.g., the

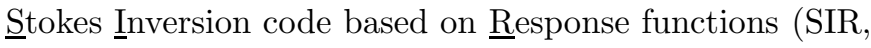
Ruiz Cobo \& del Toro Iniesta 1992) are able to reproduce the observed profiles, one has to be aware that the solution is not unique and that the solution retrieved with SIR give a magnetic field strength that increases with height which is incompatible with any theoretical model (Martínez Pillet 2000). To our believe, this is due to the inability of SIR to deal with discontinuities along the LOS. Therefore instead of trying to directly invert our observed profiles we rather elaborate on the effects that discontinuities have on the line profile. Synthesizing lines with simple geometrical models give a handle to explain qualitatively certain characteristics of the observed profiles.

From theoretical models and observations one expects that velocity gradients and gradients in the magnetic field are not smooth, as, e.g., assumed by SL92, but that discontinuities arise when the LOS crosses a flow channel. Discontinuities have previously been assumed (Solanki \& Montavon 1993; Martínez Pillet 2000) to explain the net circular polarization that is observed in sunspots.

Here, we elaborate on consequences of flow channels on the shape of Stokes profiles, rather than on the net circular polarization. In particular, we concentrate on the amplitude difference of $V$-profiles and its sign reversal in the center-side penumbra (cf. Sect. 4.1), and on abnormal $V$-profiles along the magnetic neutral line in the limb-side penumbra (cf. Sect. 4.2.2). Note that our discussion on synthetic profiles is limited to $V$-profiles. Observed characteristics of $L$ are discussed on the grounds of geometric arguments in Sect. 5.4.

The moving tube model (Schlichenmaier et al. 1998b) gives a consistent picture for the penumbral fine structure in terms of evolving flux tubes. Motivated by this model, we base our investigation on the following simplified picture of the penumbra. We deal with three different components: (1) The background magnetic field with zero velocity. The corresponding strength within the penumbra decreases radially outwards, while the inclination with respect to the surface normal increases. (2) Upflow channels, predominantly in the inner penumbra, that are hotter 
than the surroundings, optically thick, and that have a smaller magnetic field strength. (3) Radial, roughly horizontal outflows that should dominate the fine structure in the outer penumbra. These outflow channels are in temperature equilibrium with the surroundings and have approximately the same magnetic field strength as the surroundings. The inclination of the magnetic field vector within both kinds of flow channels differs from the corresponding inclination of the background magnetic field. This is the essential ingredient that potentially produces line asymmetries.

In order to isolate and understand the effects of the discontinuities that are produced by a flow channel, we consider only line profiles that stem from one single LOS. Correspondingly, we do not perform a mixing of different components which is typically done to account for lacking spatial resolution and for stray-light effects. Since such mixing is always present, we cannot expect to reproduce the exact shapes of the observed profiles, but we expect to reproduce some of the qualitative features.

The synthetic lines are obtained by solving the radiative transfer equation (Unno-Rachkovsky equations) using the code described in Grossmann-Doerth et al. (1988). Atomic data for our absorption line are taken from Zayer et al. (1989). For the penumbral atmosphere we use the model from Kjeldseth-Moe \& Maltby (1969) and further we assume that the magnetic field decreases with height according to

$B(\tau)=B_{\mathrm{c}}+50 \cdot \log \tau$,

with $B_{\mathrm{c}}$ being the magnetic field strength at optical depth unity $(\log \tau=0)$. We use a micro-turbulence velocity of $1.5 \mathrm{~km} \mathrm{~s}^{-1}$. Our synthetic lines do not contain broadening effects due to macro-turbulence or instrumental broadening. For the considerations of this section, we use a heliocentric angle of $\theta=30^{\circ}$.

\subsection{Synthetic V-profiles on center-side penumbra}

\subsubsection{Imprints of an outflow channel}

When a horizontal outflow channel in temperature equilibrium with the surroundings is placed in the photosphere, two discontinuities along the LOS are created which potentially produce asymmetries of Stokes profiles. In the upper right panel of Fig. 9 we sketch the situation that we envisage for the outer center-side penumbra. Motivated by the moving tube model (Schlichenmaier et al. 1998b), we assume a horizontal flow channel with an absolute flow velocity of $14 \mathrm{~km} \mathrm{~s}^{-1}$ which is placed in the deep photosphere, $-0.35 \leq \log \tau \leq-0.65$, being embedded in a background magnetic field with an inclination of $70^{\circ}$ (cf. right part of the upper right panel). The magnetic field strength at continuum height is taken to be $B_{\mathrm{c}}=0.12 \mathrm{~T}$ (1200 Gauss), and decreases upwards as given by Eq. (10) (left part of that panel). Note that the flow channel does not introduce a fluctuation of the magnetic field strength. The left panels of Fig. 9 shows the magnetic field strength,
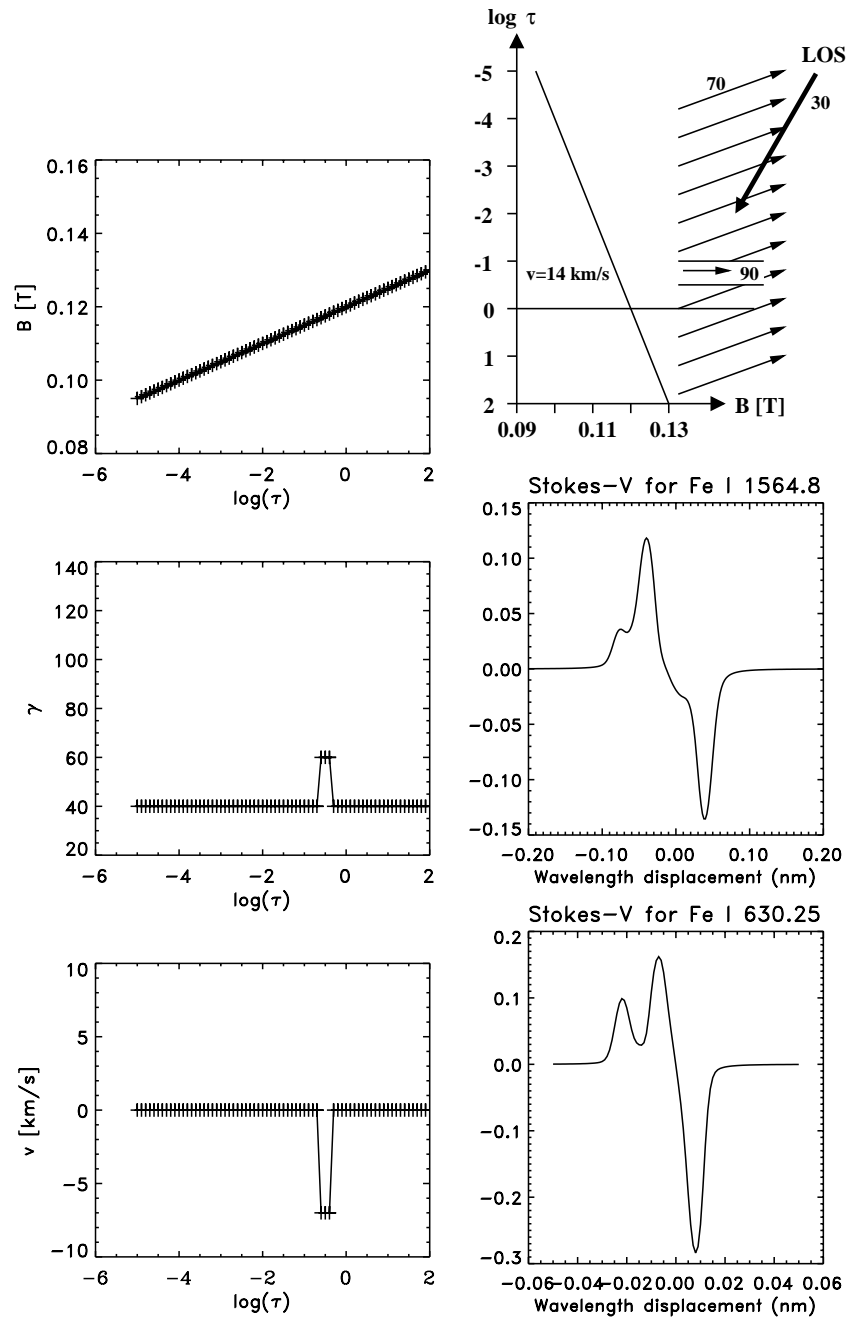

Fig. 9. Model configuration for the outer center-side penumbra. The upper right panel sketches the geometry: the right portion depicts the location of the flow channel in $\log \tau$ and the angles of the LOS, the background magnetic field, and the flow channel. The decrease of the magnetic field strength with $\log \tau$ is depicted in the left portion of this panel. The left column shows the variation of $B, \gamma$, and the LOS-component of $v$ along the LOS, plotted versus $\log \tau$. The middle right and lower right panel depict the resulting $V$-profiles of Fe I 1564.8 and Fe I630.25, respectively.

$B$ (top), the angle of the magnetic field vector relative to the LOS, $\gamma$ (middle), changing from $40^{\circ}$ to $60^{\circ}$, and the LOS-component of the flow velocity, $v$ (bottom), along the LOS as function of $\log \tau$. The latter two quantities, $\gamma$ and $v$, display a discontinuity at the location of the flow channel.

This configuration results in a $V$-profile (middle right panel in Fig. 9) that shows an amplitude difference of $\mathrm{d} a=-1.7 \%$ for the infrared line at $1564.8 \mathrm{~nm}$, reproducing the correct sign of our observed value. We note that the sign and the value of the amplitude difference cannot be predicted by simple arguments based on the gradients of the magnetic field and the velocity. Such arguments (e.g., Solanki \& Pahlke 1988) are only valid for the sign of the area asymmetry. 
Generally speaking, the resulting profile can be understood as a superposition of two components: an unshifted component stemming from the background and a blue-shifted component from the flow channel, which one might consider as a line satellite. The amplitude difference is produced by the interference of the main component and the blue-shifted component. The degree of asymmetry is sensibly influenced by the relative weights of the two components.

For a very thin channel, e.g., $-0.55<\log \tau<-0.45$, the main component dominates the $V$-profile and, consequently, the asymmetry is rather small, $\mathrm{d} a=-0.6 \%$. Increasing the thickness of the flow channel, $-1.05<$ $\log \tau<-0.45$, leads to a larger asymmetry, $\mathrm{d} a=-2.2 \%$. Further increasing the thickness such that the flow channel and the background become of comparable strengths leads to a decrease of the amplitude difference. For a flow channel with $-1.75<\log \tau<-0.45$, the amplitude difference becomes positive.

\subsubsection{Imprints of a hot upflow}

We model the situation of a hot, optically thick upflow in the inner penumbra placing it in deep photospheric layers. The moving tube model predicts the magnetic field strength inside the hot part of the tube to be substantially lower than in the background. This is a consequence of the increased gas pressure and the requirement of pressure balance. For our simple line synthesis calculations we take $0.16 \mathrm{~T}$ in the background and $0.10 \mathrm{~T}$ for the tube. The inclination of the background field is assumed to be a little steeper than in the outer penumbra, namely $60^{\circ}$. For simplicity, the inclination of the upflow is chosen to be the same as for the background field, but we have convinced ourselves that the results for steeper upflows, e.g., with an inclination of $30^{\circ}$, are almost identical. The velocity of the flow is taken from the moving tube model to be $4 \mathrm{~km} \mathrm{~s}^{-1}$. The geometrical situation is sketched in Fig. 10. Figure 10 shows the resulting $V$-profile. It exhibits a positive amplitude difference, $\mathrm{d} a=2.1 \%$, which value and sign compares well with our observed profiles in the inner center-side penumbra.

Thickness of upflow: If the upflow extends higher up into the atmosphere, then the amplitude difference increases and for an upflow between, say, $2<\log \tau<-1$, we obtain $\mathrm{d} a=3.9 \%$. If the upflow is only present in very deep layers, $\mathrm{d} a$ decreases, and reaches $\mathrm{d} a=0.4 \%$ for $2<\log \tau<-0.05$.

Result for Fel 630.25: As mentioned in Sect. 4.1, measurements in the iron triplet $(g=2.5)$ at $630.25 \mathrm{~nm}$ yield negative values for the amplitude difference throughout the penumbra (SL92). Using our models for the inner and outer penumbra, and performing the calculation for this line, we indeed find that $\mathrm{d} a$ is negative in both cases. The corresponding profiles with $\mathrm{d} a=-12 \%$ and $\mathrm{d} a=-7 \%$
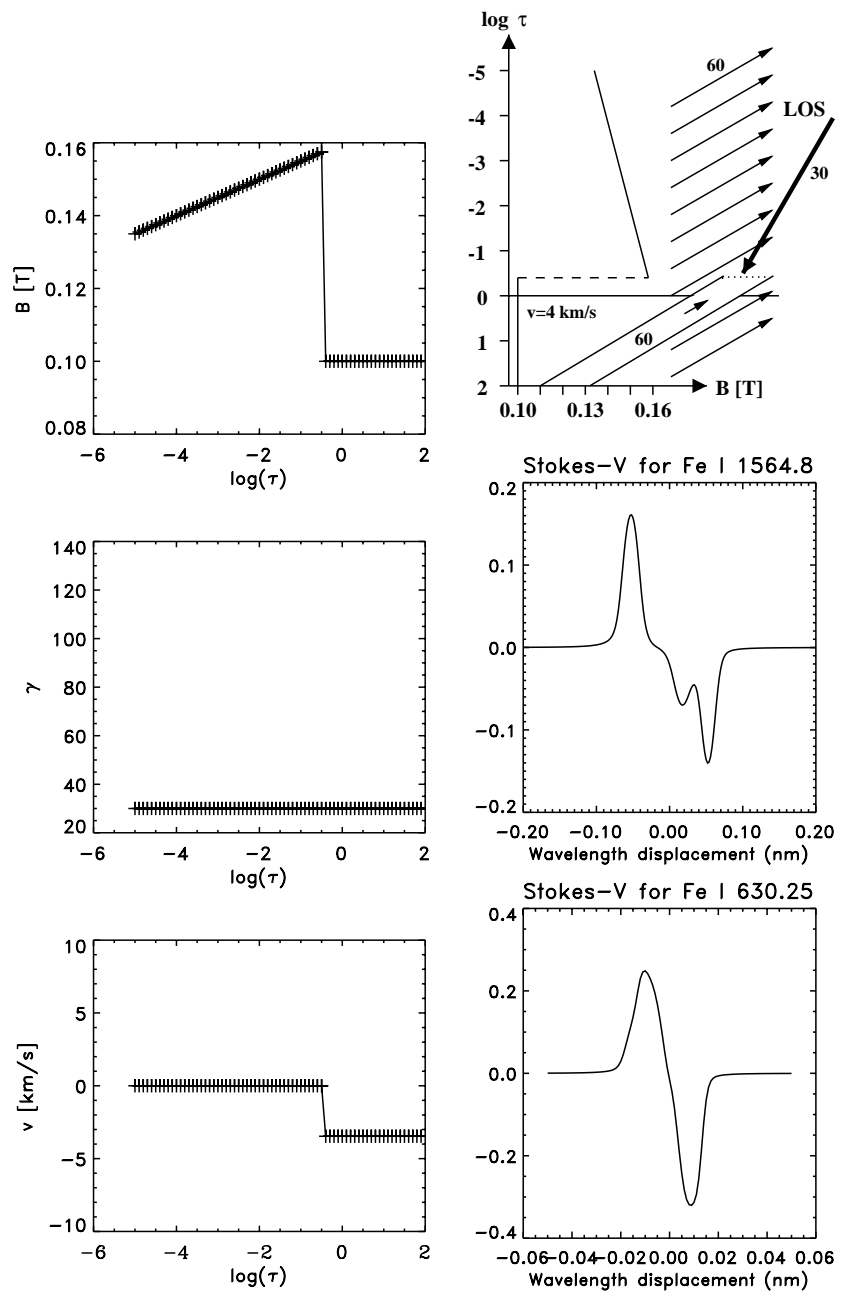

Fig. 10. Model configuration of the inner center-side penumbra (same as Fig. 9). A hot, optically thick upflow exhibiting a reduced magnetic field strength is located in the deep photosphere.

are shown in the lower right panels of Figs. 9 and 10, respectively. Hence our simple model is capable to reproduce observed values for $\mathrm{d} a$ in two quite different photospheric absorption lines.

\subsubsection{Sign reversal of the amplitude difference}

Our synthetic line calculations show that the sign reversal of the amplitude difference in $V$-profiles of Fe I 1564.8 along a radial cut through the center-side penumbra can be reproduced, if one assumes that upflows dominate the inner while outflows dominate the outer penumbra. This picture is further supported by the following argument: since the penumbral brightness does only slightly depend on the radial distance from spot center, hot upflow channels should be uniformly distributed across the penumbra. These upflow channels are the source of the roughly horizontal radial outflow, in which the plasma radiatively cools off to temperatures of the surroundings and becomes transparent. If each upflow channel produces a radial outflow extending from it's upflow source to the outer 

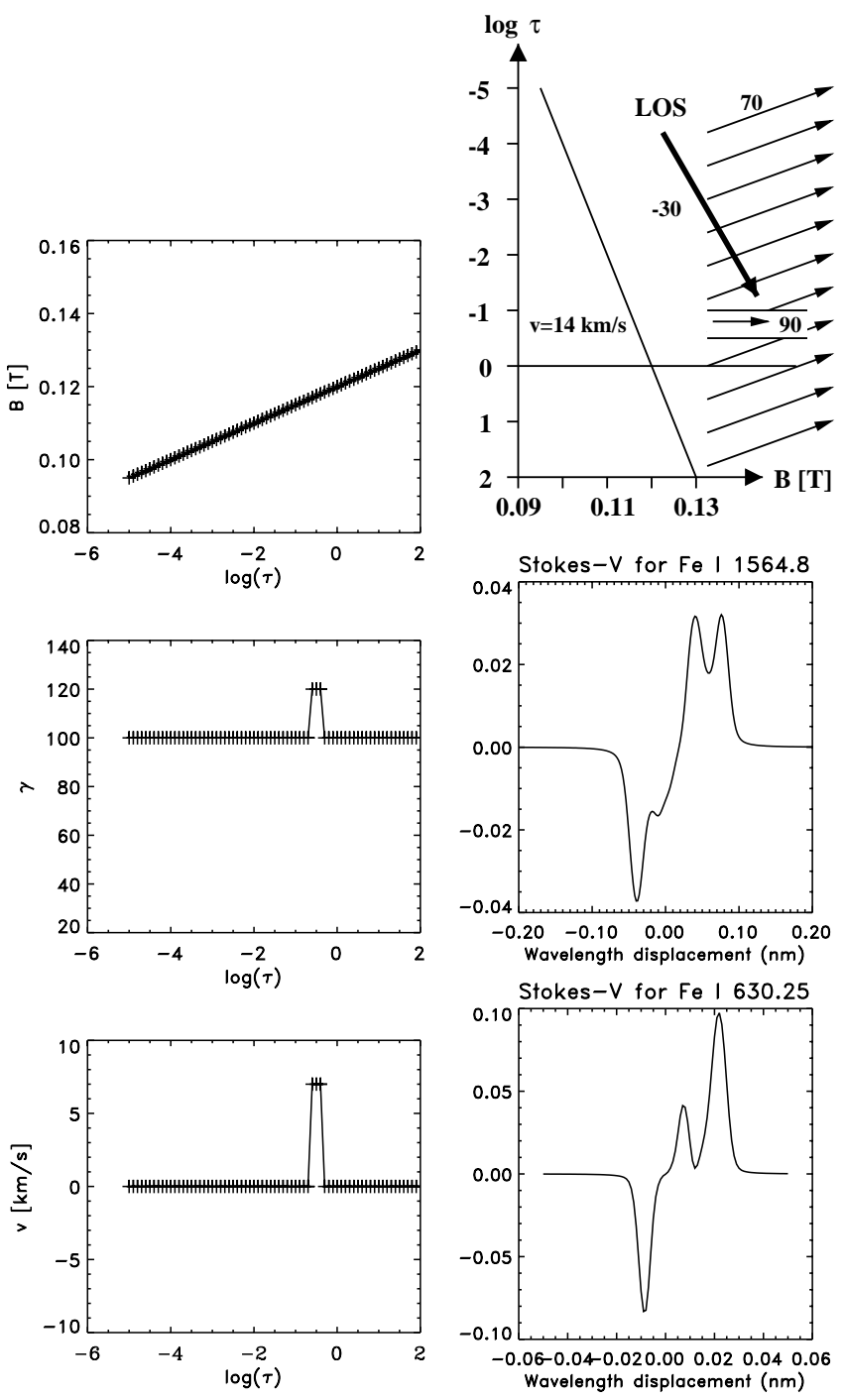

Fig. 11. Model configuration for the outer limb side penumbra (same as Fig. 9).

penumbra, a high number of outflow channels should exist in the outer penumbra, while only a few outflow channels are present in the inner penumbra. This situation naturally leads to the above postulated predominance of upflows in the inner and outflows in the outer penumbra and consequently to the observed sign reversal in the amplitude difference of the infrared iron triplet at $1564.8 \mathrm{~nm}$.

It is interesting to note that the sign reversal is not observed in FeI630.25 and that the synthetic lines based on the geometry of Figs. 10 and 9 for that absorption line also do not show a sign reversal of the amplitude difference. In contrast to the infrared line, the amplitude difference for Fe I630.25 is negative in both cases, just as it is observed by SL92.

\subsection{Synthetic V-profile for outer limb-side penumbra}

The maps of the amplitude difference, $\mathrm{d} a$, in Fig. 2 reveal that $\mathrm{d} a$ becomes negative beyond the MNL in the outer limb-side penumbra (cf. list item 3 of Sect. 4.2.1).
We model this situation by using the same configuration as for the outer center-side penumbra, except that we use a different angle of the LOS in order to account for a limb-side penumbra (Fig. 11). And indeed, the resulting $V$-profile (middle right panel of Fig. 11) for the infrared line shows a negative amplitude difference, just as it is observed. The spot that is discussed in SL92 has its MNL at the very outer penumbra, such that we cannot compare synthetic profiles beyond the MNL with their data.

\subsubsection{Abnormal V-profile in outer limb-side penumbra}

In list item 2 of Sect. 4.2.1 we state that abnormal $V$-profiles, in this case abnormal blue lobes of positive polarity, exist throughout the penumbra for small heliocentric angles (cf. $\theta=33^{\circ}$ in Fig. 6), and that they are present only in the vicinity of the MNL for a larger $\theta$ (cf. $\theta=59^{\circ}$ in Fig. 5). We surmise that upflow channels are responsible for this finding, based on the assumption that upflow channels have an inclination between $30^{\circ}$ and $50^{\circ}$ : for $\theta=33^{\circ}$ such an upflow channel would have the opposite polarity than the horizontal flow channels and would produce a blue-shifted positive blue lobe in the $V$-signal, similar to the situation in Fig. 6. For $\theta=59^{\circ}$ such an upflow channel has the same polarity as the horizontal outflows and cannot produce a blue-shifted component, in conformance with observations.

\subsection{Modeling profiles along the magnetic neutral line}

In Sect. 4.2.2 $V$-profiles along the magnetic neutral line are displayed which have abnormal shapes, i.e, three or four lobes. In this section we aim to demonstrate that such abnormal $V$-profiles can in principle be reproduced with synthetic profiles of only one LOS, i.e., with a model where the line asymmetry is produced solely by discontinuities along the LOS. In particular let us concentrate on the three-lobe $V$-profiles of Fig. 7. Here the MNL is in the middle of the penumbra, and we expect that upflows and horizontal outflows (stemming from upflow sources located further inwards) may contribute to the profiles. We simulate this configuration by putting an optically thick upflow in the deepest layers and by stacking a horizontal outflow just above it. The background magnetic field is assumed to be perpendicular to the LOS, such that the $V$-profile exclusively stems from the up- and outflow, since only there the inclination of the magnetic field has a component along the LOS. The geometrical set-up is sketched in the upper right panel of Fig. 12 and the resulting synthetic $V$-profiles are shown in the middle right panel for the infrared line and in the lower right panel for the visible line.

It is seen that such a geometrical configuration, which is motivated by the moving-tube model, captures the characteristics of the observed abnormal $V$-profiles, for both, the infrared and visible line. This includes that the amplitude asymmetry of the infrared synthetic $V$-profile is 

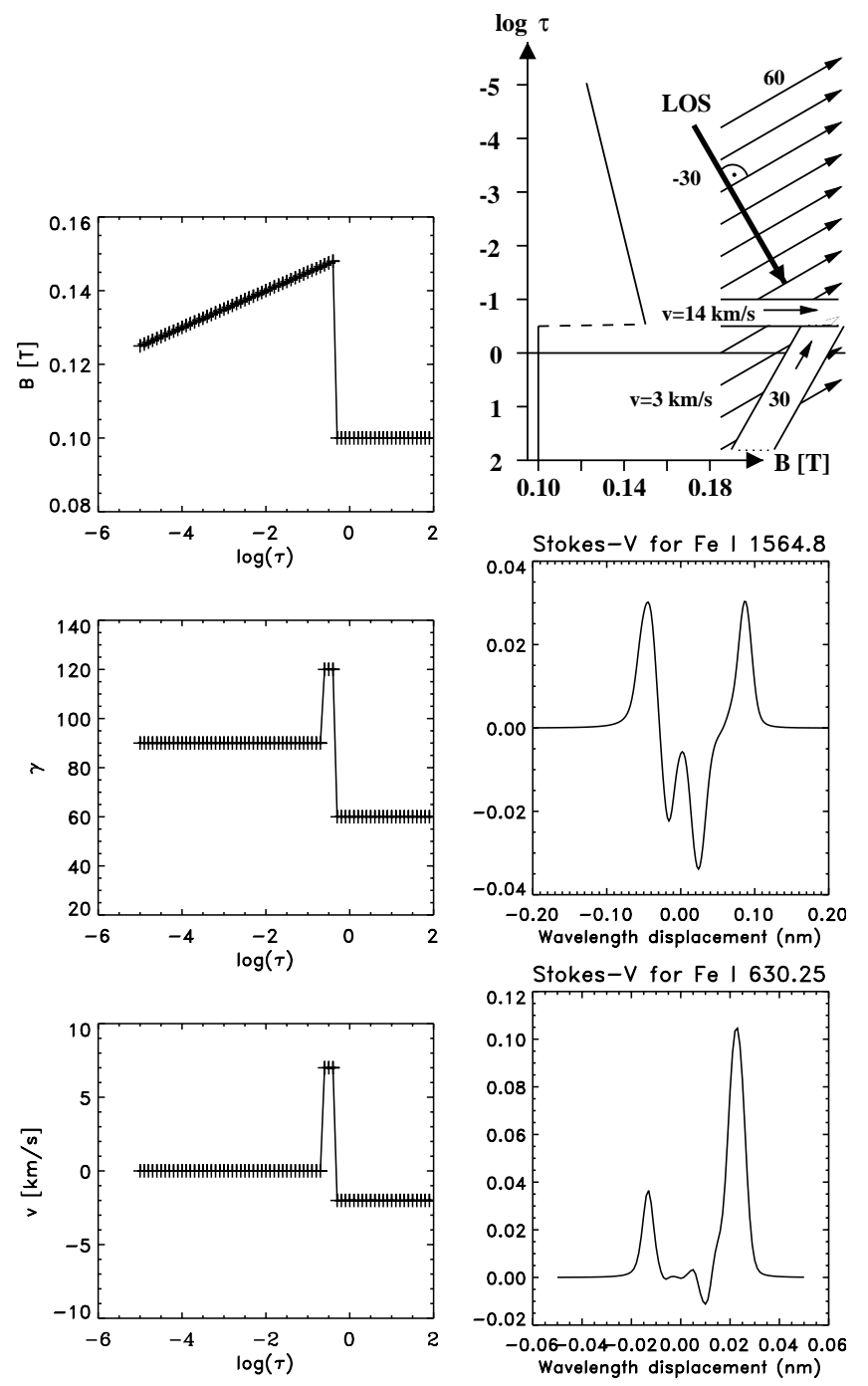

Fig. 12. Model configuration for the magnetic neutral line (same as Fig. 9).

negative, as it is observed (cf. Figs. 2 and 7). The essential ingredient to produce such abnormal $V$-profiles is the presence of magnetic components of different polarities: The upflow has a LOS-component of the magnetic field towards the observer, while the horizontal outflow has a LOS-component away from the observer. These two components along the LOS have different magnetic fields strengths, i.e., produce a different splitting of the corresponding $\sigma$-components, and, most important, they are Doppler-shifted with respect to each other.

\subsection{Discussion of the observed $\pi$-component of $L(\lambda)$}

\subsubsection{Observed splitting and asymmetry of the $\pi$-component}

In Sect. 4.1 we report that we directly measure a Dopplershift of $5 \mathrm{~km} \mathrm{~s}^{-1}$ between the two $\pi$-components in the mid center-side penumbra for the spot at $\theta=56^{\circ}$. Obviously, the line profile is a superposition of two components that are Doppler-shifted against each other. The fact that we do not observe this splitting closer to disk center (cf. Fig. 4) indicates that the (Evershed) flow is predominantly horizontal, since then the LOS-component of the flow velocity is correspondingly lower.

On the limb-side penumbra, the $\pi$-component of $L$ displays the typical asymmetry property of the Evershed flow (wing stronger shifted than the core), cf. Figs. 5-7 in Sect. 4.2. The fact that we observe a splitting, i.e., two distinguished components, on the center-side and only an asymmetry on the limb side can be ascribed to the relative weights of flow channel and background magnetic field: a horizontal flow channel contributes equally to $L$ on both sides of the penumbra, while on the center-side the background magnetic field is mainly in the direction of the LOS leading to a smaller contribution to $L$ than on the limb side. Therefore the imprints of the flow channel in $L$ may lead to a splitting on the center side, while the component of the flow channel on the limb side is small compared to the background component.

The phenomenon of splitting and asymmetry in the $\pi$-component of $L$ is related to the presence of line asymmetries in Stokes- $I$ profiles of magnetically insensitive lines (see Sect. 1). We note that it is not clear a priori whether the two components lie next to each other in lateral direction and are spatially unresolved or whether they are stacked in vertical direction and are not resolved in depth by our absorption line.

\subsubsection{Doppler shift between $V$ and $\pi$-component}

It seems puzzling that the center of the $\pi$-component of $L$ is blue-shifted relative to the zero-crossing of the $V$-profile in both the outer center-side and the outer limbside penumbra (cf. Figs. 3-6). This seems to contradict the fact that the profiles are red-shifted on the limb side and blue-shifted on the center side. However, as in the preceeding subsection, one has to be aware that a horizontal flow channel contributes equally to the line profiles on both sides of the penumbra, while the background contribution for $L$ is much larger on the limb side (magnetic field predominantly perpendicular to the LOS) than on the center side (magnetic field predominantly in the direction of the LOS), and vice versa for $V$. On the center-side the Stokes- $V$ profile is dominated by the non-moving background component, such that the $V$-profile there is mainly unshifted, while the $\pi$-component of $L$ is sensible to the blue shift due to the flow channel. Hence the $\pi$-component should be blue-shifted relative to the $V$-profile on the center-side penumbra. For the limb-side penumbra the argument is reverses. The background magnetic field dominates the $L$-signal and leads to a mainly unshifted profile for $L$, while the red shift due to the flow channel is clearly visible on the $V$-profile. Hence the $\pi$-component should also be blue-shifted relative to the $V$-profile on the limb side. We note that it is essential for the upper argument to assume that magnetic flux tubes that carry the flow 
have a different inclination than the background magnetic field, which we assume to be non-moving.

\section{Summary and conclusions}

We present measurements of penumbral Stokes profiles in the neutral iron triplet $(g=3)$ at $1564.8 \mathrm{~nm}$. Our data set encloses 3 different round sunspots at various heliocentric angles. As a measure for the asymmetry of $V$-profiles we introduce the amplitude difference, $\mathrm{d} a$, and the net circular polarization, NCP. We produce maps of these quantities and discuss their spatial dependence and the corresponding shapes of the Stokes profiles within penumbrae at different heliocentric angles. Summarizing Sect. 4 and taking into account measurements in Fe I 630.25 (Sanchez Almeida \& Lites 1992; Westendorp Plaza et al. 2001), we find the following peculiarities for the shapes of Stokes- $V$ :

1. In the outer center-side penumbra $\mathrm{d} a$ is negative, while it is positive in the inner center-side penumbra. Measurements in FeI630.25 by SL92 yield that $\mathrm{d} a$ is negative throughout the the center-side penumbra (cf. item 1 in Sect. 4.1).

2. In the outer limb-side penumbra, beyond the magnetic neutral line $\mathrm{d} a$ is positive for Fe I 1564.8 (cf. item 3 in Sect. 4.2.1).

3. Along the magnetic neutral line $V$-profiles typically have three or more lobes. This is the case for both lines. For small heliocentric angle abnormal profiles are also seen beyond the magnetic neutral line in the outer penumbra (cf. item 2 in Sect. 4.2.1 and item 1 in Sect. 4.2.2).

4. The NCP maps for Fe I 1564.8 are highly antisymmetric with respect to the axis-of-symmetry, while corresponding maps for FeI630.25 are symmetric (cf. Sect. 4.3).

For the profiles of linearly polarized light we find:

5 . On the center-side penumbra at a heliocentric angle of $56^{\circ}$ a Doppler-shift as high as $5 \mathrm{~km} \mathrm{~s}^{-1}$ can be directly measured by the splitting of the $\pi$-components of the linearly polarized component (cf. item 2 in Sect. 4.1).

6. In limb-side penumbrae, the profiles of the $\pi$-component show the typical asymmetry properties of the Evershed flow as observed in Stokes- $I$ of magnetically insensitive lines (cf. item 4 in Sect. 4.2.1 and item 2 in Sect. 4.2.2).

7. In the outer center- and limb-side penumbrae the center of the $\pi$-component is blue-shifted relative to the zero-crossing of the $V$-profile (cf. item 3 in Sect. 4.1 and item 4 in Sect. 4.2.1).

Obviously, penumbral Stokes profiles have complex shapes which often change at small scales (from pixel to pixel) within the penumbra, and which show different characteristics for different heliocentric angles and for different absorption lines. Nevertheless, we demonstrate in Sect. 5 that their large variety can be qualitatively reproduced by a simple model for the penumbra which is motivated by the more sophisticated moving tube model of Schlichenmaier et al. (1998b).

This model consists of simplified model atmospheres which include a flow channel in deep photospheric layers. The synthetic profiles are computed for Fe I 1564.8 and for Fe I 630.25, taking into account only a single LOS instead of introducing a multi-component model. We envisage the penumbra to be composed of hot upflow channels, cool outflow channels, and a non-moving background magnetic field that carries most of the magnetic flux. Upflows and outflows are present everywhere in the penumbra, but as we discuss in Sect. 5.1.3, they should be distributed such that hot upflows dominate in the inner penumbra, and cool outflows dominate in the outer penumbra. With these assumptions, we can reproduce qualitatively the observational findings (1), (2), and (3) of the upper list. We mention that point (4) of the upper list can be reconciled with a similar, but more sophisticated model by Müller (2001).

We propose an explanation for the points (5), (6), and (7) of the upper list in Sect. 5.4, based on the assumption that the flow is contained in mostly horizontal flux tubes which are embedded in a background magnetic field of different inclination.

From these results we conclude that the moving tube model captures the essential features that are necessary to retrieve synthetic profiles that are consistent with a large variety of measurements in two different photospheric lines.

Acknowledgements. We thank D. Soltau and O.v.d. Lühe for their help planing and performing the observation campaign in November 1999, as well as the technical staff of the VTT for assisting the observations. We are indebted to U. GrossmannDoerth for providing the code DIAMAG to synthesize profiles. R.S. gratefully acknowledges the support by the Deutsche Forschungsgemeinschaft, DFG (LU 706/1-1). Part of this work has been funded by the Spanish DGES (project PB 95-0028C). Thanks are also due to O. Steiner for critical comments on the manuscript.

\section{References}

Ballesteros, E., Collados, M., Bonet, J. A., et al. 1996, A\&AS, 115,353

Balthasar, H., Schmidt, W., \& Wiehr, E. 1997, Sol. Phys., 171, 331

Beckers, J. M., \& Schröter, E. H. 1969, Sol. Phys., 10, 384

Bumba, V. 1963, Bulletin of the Astronomical Institutes of Czechoslovakia, 14, 137

Capitani, C., Landi degl'Innocenti, E., Cavallini, F., Ceppatelli, G., \& Landi degl'Innocenti, M. 1989, Sol. Phys., 120,173

Collados, M. 1999, in ASP Conf. Ser. 184, Third Advances in Solar Physics Euroconference: Magnetic Fields and Oscillations, 3

Degenhardt, D., \& Wiehr, E. 1994, A\&A, 287, 620

Evershed, J. 1909, MNRAS, 69, 454

Grigorjev, V. M., \& Katz, J. M. 1972, Sol. Phys., 22, 119 
Grossmann-Doerth, U., Larsson, B., \& Solanki, S. K. 1988, Rüedi, I., Solanki, S. K., Keller, C. U., \& Frutiger, C. 1998, A\&A, 204, 266

Holmes, J. 1963, MNRAS, 126, 155

Ichimoto, K. 1987, PASJ, 39, 329

Ichimoto, K. 1988, PASJ, 40, 103

Kjeldseth-Moe, O. 1967, in IAU Symp. 35, Structure and development of solar active regions, ed. K. O. Kiepenheuer, 202

Kjeldseth-Moe, O., \& Maltby, P. 1969, Sol. Phys., 8, 275

Landolfi, M., \& Landi degl'Innocenti, E. 1996, Sol. Phys., 164, 191

Lide, D. 1993, CRC Handbook of Chemistry and Physics

Makita, M. 1979, PASJ, 31, 575

Maltby, P. 1964, Astrophysica Norvegica, 8, 205

Martínez Pillet, V. 2000, A\&A, 361, 734

Martínez Pillet, V., Collados, M., Sánchez Almeida, J., et al. 1999, in ASP Conf. Ser. 183, High Resolution Solar Physics: Theory, Observations, and Techniques, 264

Müller, D. 2001, Diploma Thesis, University of Freiburg

Rimmele, T. R. 1995, A\&A, 298, 260 A\&A, 338, 1089

Ruiz Cobo, B., \& del Toro Iniesta, J. C. 1992, ApJ, 398, 375

Sanchez Almeida, J., \& Lites, B. W. 1992, ApJ, 398, 359

Schlichenmaier, R., Jahn, K., \& Schmidt, H. U. 1998a, ApJ, 493, L121

Schlichenmaier, R., Jahn, K., \& Schmidt, H. U. 1998b, A\&A, 337,897

Schmidt, W., \& Kentischer, T. 1995, A\&AS, 113, 363

Schröter, E. H. 1965, Z. Astrophys., 62, 228

Servajean, R. 1961, Annales d'Astrophysique, 24, 1

Solanki, S. K., \& Montavon, C. A. P. 1993, A\&A, 275, 283

Solanki, S. K., \& Pahlke, K. D. 1988, A\&A, 201, 143

St. John, C. E. 1913, ApJ, 37, 322

Stellmacher, G., \& Wiehr, E. 1980, A\&A, 82, 157

Westendorp Plaza, C., del Toro Iniesta, J. C., Ruiz Cobo, B., et al. 2001, ApJ, 547, 1148

Wiehr, E. 1995, A\&A, 298, L17

Zayer, I., Solanki, S. K., \& Stenflo, J. O. 1989, A\&A, 211, 463 